\title{
Carotid-Sparing TomoHelical 3-Dimensional Conformal Radiotherapy for Early Glottic Cancer
}

\author{
Chae-Seon Hong, $\mathrm{PhD}^{1}$ \\ Dongryul Oh, MD ${ }^{1}$ \\ Sang Gyu Ju, $\mathrm{PhD}^{1}$ \\ Yong Chan Ahn, MD, PhD' \\ Jae Myoung Noh, $\mathrm{MD}^{1}$ \\ Kwangzoo Chung, $\mathrm{PhD}^{1}$ \\ Jin Sung Kim, $P h D^{1}$ \\ Tae-Suk Suh, $\mathrm{PhD}{ }^{2}$
}

\section{${ }^{1}$ Department of Radiation Oncology, Samsung Medical Center, Sungkyunkwan University School of Medicine, Seoul, \\ ${ }^{2}$ Department of Biomedical Engineering and Research Institute of Biomedical Engineering, The Catholic University of Korea, Seoul, Korea \\ Correspondence: Sang Gyu Ju, PhD \\ Department of Radiation Oncology, \\ Samsung Medical Center, 81 Irwon-ro, \\ Gangnam-gu, Seoul 06351, Korea \\ Tel: 82-2-3410-2612 \\ Fax: 82-2-3410-2619 \\ E-mail: sg.ju@samsung.com}

Co-Correspondence: Yong Chan Ahn, MD, PhD, Department of Radiation Oncology,

Samsung Medical Center, 81 Irwon-ro,

Gangnam-gu, Seoul 06351, Korea

Tel: 82-2-3410-2612

Fax: 82-2-3410-2619

E-mail: ahnyc@skku.edu

Received September 21, 2014

Accepted December 24, 2014

Published Online March 6, 2015

${ }^{*}$ Chae-Seon Hong and Dongryul Oh contributed equally to this work.

\section{Purpose}

The purpose of this study was to investigate the dosimetric benefits and treatment efficiency of carotid-sparing TomoHelical 3-dimensional conformal radiotherapy (TH-3DCRT) for early glottic cancer.

\section{Materials and Methods}

Ten early-stage (T1NOMO) glottic squamous cell carcinoma patients were simulated, based on computed tomography scans. Two-field 3DCRT (2F-3DCRT), 3-field intensity-modulated radiation therapy (3F-IMRT), TomoHelical-IMRT (TH-IMRT), and TH-3DCRT plans were generated with a 67.5-Gy total prescription dose to the planning target volume (PTV) for each patient. In order to evaluate the plan quality, dosimetric characteristics were compared in terms of conformity index $(\mathrm{Cl})$ and homogeneity index $(\mathrm{HI})$ for PTV, dose to the carotid arteries, and maximum dose to the spinal cord. Treatment planning and delivery times were compared to evaluate treatment efficiency.

\section{Results}

The median $\mathrm{Cl}$ was substantially better for the 3F-IMRT (0.65), TH-IMRT (0.64), and TH-3DCRT (0.63) plans, compared to the 2F-3DCRT plan (0.32). PTV HI was slightly better for TH-3DCRT and TH-IMRT (1.05) compared to 2F-3DCRT (1.06) and 3F-IMRT (1.09). TH-3DCRT, 3F-IMRT, and TH-IMRT showed an excellent carotid sparing capability compared to 2F-3DCRT $(p<0.05)$. For all plans, the maximum dose to the spinal cord was < 45 Gy. The median treatment planning times for 2F-3DCRT (5.85 minutes) and TH-3DCRT (7.10 minutes) were much lower than those for 3F-IMRT (45.48 minutes) and TH-IMRT (35.30 minutes). The delivery times for 2F-3DCRT (2.06 minutes) and 3F-IMRT (2.48 minutes) were slightly lower than those for TH-IMRT (2.90 minutes) and TH-3DCRT (2.86 minutes).

\section{Conclusion}

TH-3DCRT showed excellent carotid-sparing capability, while offering high efficiency and maintaining good PTV coverage.

\section{Introduction}

Early glottic cancer (EGC) is a highly curable malignancy. Radiation therapy (RT) has been established as the primary

\author{
Key words \\ Early glottic cancer, Carotid sparing, \\ TomoHelical three-dimensional conformal radiotherapy, \\ Intensity-modulated radiotherapy
}


left and right of the target volume (TV). As a result, increased incidences of stroke and other cerebrovascular events have been reported in patients who have survived over 10 years since undergoing the treatment for EGC [3-5]. In particular, the risk of ischemic stroke increases 10-fold in patients undergoing traditional RT for EGC if younger than 60 years of age [5].

For these reasons, various treatment techniques have been introduced to decrease the dose administered to CA during RT for EGC [6-9]. Intensity-modulated radiation therapy (IMRT) can maintain good coverage while significantly reducing the dose administered to the $\mathrm{CA}$, as compared with traditional LOF and 3-dimensional conformal RT (3DCRT) [6-8]. Further, Chatterjee et al. [9] have reported that helical tomotherapy-based IMRT offers significant improvements to CA sparing. Inverse planning-based IMRT is highly effective in reducing the dose to adjacent organ at risks (OAR) and improves target dose conformity by controlling the beam intensity. However, as compared with 3DCRT, IMRT involves complicated treatment plans that are closely related to poor treatment efficiency, as well as to long planning and treatment times. The complicated plans also require additional pretreatment quality assurance in busy clinics [10]. Furthermore, as compared with 3DCRT, these more complicated plans are associated with higher risks of error. In particular, IMRT is associated with higher risk of inaccurate dose delivery to moving targets, which results from the interplay between multi-leaf-collimator (MLC) and organ motions [11]. Because the TV is relatively small in cases of EGC, it is more likely to be influenced by movement during IMRT, and therefore requires careful consideration $[12,13]$.

Tomotherapy options can be classified as either IMRT or 3DCRT, as performed using either a helical mode or a direct mode (fixed beams), depending on the dose planning and beam delivery technique. TomoHelical 3DCRT (TH-3DCRT) is a form of helical conformal arc therapy. It has the advantage of offering plan and beam delivery times that are generally shorter than those associated with IMRT. TH-3DCRT presents these time-advantages because it does not include an intensity-modulation process that relies upon inverse planning. Thus, TH-3DCRT can be an effective RT modality for EGC and other targets that have simple shapes and do not require complex beam-intensity modulation (because the internal beam path length is short).

Recently, researchers have used dosimetric comparison studies to identify the techniques that minimize the CA dose in RT for EGC. However, prior studies have not included clear comparisons of the dosimetric characteristics of helical tomotherapy (TH-IMRT) and linear-accelerator-based IMRT. Further, prior comparison studies have not investigated TH-3DCRT for EGC. In the present study, we compared 3DCRT, IMRT, TH-IMRT, and TH-3DCRT in terms of their dosimetric characteristics and treatment time efficiencies for EGC. By performing this comparison, we aimed to evaluate the performance of various treatment techniques in clinical application.

\section{Materials and Methods}

\section{Patient selection and definition of the TV}

Between February 2013 and December 2014, a total of 21 patients with early glottis carcinoma (cT1-2N0M0) were treated with definitive RT. We selected 10 of these patients for this study, each of whom had T1N0 glottic squamous cell carcinoma and underwent curative RT with daily imageguidance. All patients underwent computed tomography (CT) (2.5-mm slice thickness; LightSpeed, GE Healthcare, Milwaukee, WI) after being immobilized in supine position, using thermoplastic masks. Each patient's CT image dataset was transferred to the treatment planning system (TPS) (Pinnacle $^{3}$ ver. 9.2, Philips Medical System, Madison, WI), and the clinical target volume (CTV), planning target volume (PTV), spinal cord, and both CAs were delineated. The CTV was delineated based on the traditional $5 \times 5 \mathrm{~cm}^{2}$ field size. The beam isocenter was placed at the level of the true vocal cords [6]. The CTV was contoured as follows: superiorly, at the thyroid notch or at the most cranial extent of arytenoid cartilage; inferiorly, at 1-1.5 cm below the level of the true vocal cord; posteriorly, the CTV included arytenoid cartilage and posterior commissure; and anterolaterally, the CTV was drawn along the inner portion of the thyroid cartilage and encompassed the anterior commissure. To create the PTV, the CTV was expanded by $3 \mathrm{~mm}$ in the lateral and anterior directions and by $1 \mathrm{~mm}$ in the posterior direction. A lesser expansion was used in the posterior direction because we sought to reduce the irradiated dose to the posterior pharyngeal wall. With regard to clinical structures, the CAs and the spinal cord were delineated. Such OAR volumes were delineated up to 2-cm superiorly and inferiorly beyond PTV.

\section{Treatment planning}

For each patient, four sets of treatment plans were created to analyze the dosimetric characteristics and treatment time efficiency: (1) 2-field 3DCRT (2F-3DCRT), (2) 3-field IMRT (3F-IMRT), (3) TH-IMRT, and (4) TH-3DCRT. 2F-3DCRT and 3F-IMRT were planned using a Pinnacle TPS with a Clinac 6EX (Varian, Palo Alto, CA). TH-IMRT and TH-3DCRT were planned using a TomoTherapy TPS with a TomoHD (TomoTherapy, Accuray, Sunnyvale, CA). All plans involved 
Table 1. Dose constraints for inverse planning

\begin{tabular}{lc} 
Structure & Constraint \\
PTV & $\mathrm{D}_{95} \geq 67.5 \mathrm{~Gy}$ \\
Spinal cord & $\mathrm{D}_{\max } \leq 45 \mathrm{~Gy}$ \\
Carotid artery & $\mathrm{V}_{30} \leq 20 \%$ \\
& $\mathrm{~V}_{10} \leq 50 \%$ \\
\hline
\end{tabular}

PTV, planning target volume; $\mathrm{D}_{\mathrm{x}}$, dose received by $\mathrm{x} \%$ of the volume; $D_{\max }$, maximum dose; $V_{D}$, the percentage of the organ volume that received D Gy or more.

6-MV X-rays. We employed 2-mm (Pinnacle) and 1.95-mm (TomoTherapy) dose calculation grid sizes. The prescription dose was 67.5 Gy in 30 fractions (2.25 Gy per fraction) to the PTV. All plans were normalized such that $95 \%$ of the PTV received $100 \%$ of the prescription dose.

Traditional LOF was not included in this study because it generally results in a high CA dose. Instead, two wedged anterior-oblique fields (2F-3DCRT, gantry angles $70^{\circ}$ and $290^{\circ}$ ) were used to provide more effective means of reducing the CA dose. For the 3F-IMRT, an anterior field was added to the same right and left anterior oblique fields that were employed in 2F-3DCRT. The direct machine parameter optimization module, supplied by the TPS manufacturer, was used for dose optimization, which was performed via inverse planning. To provide the PTV dose constraint for inverse planning, PTV was set to meet the prescription dose (Table 1) [6]. The volumes of the CA receiving $35 \mathrm{~Gy}$ and $10 \mathrm{~Gy}$ were constrained to be below $20 \%$ and $50 \%$, respectively. Finally, the spinal cord dose was constrained to be below $45 \mathrm{~Gy}$. For each plan, we used the same number of iterations (250) during the dose optimization process. During inverse planning, the priority was given to PTV. When the PTV dose reached the constraint early, optimization was continued to reduce the OAR dose until the iteration limit, while maintaining the PTV dose.

For TH-IMRT, we used a field width of $2.5 \mathrm{~cm}$, a modulation factor of 2.0, and a pitch of 0.287 (to avoid the thread effect). TH-IMRT inverse planning involved the same number of iterations, and the same dose constraints that were specified above for 3F-IMRT. To spare the CA, a directional blocking was applied to the right and left $\mathrm{CA}$, thus disabling the primary beam if the blocked structure was proximal to the target, but not if the beamlets entered the target first. TH-3DCRT was performed under the same conditions as TH-IMRT, with the exception of intensity modulation.

\section{Comparison of dosimetric characteristics and treatment efficiency}

To quantitatively evaluate the plan quality under identical conditions, all calculated plans were transferred to the same dose evaluation software (MIM Maestro, MIM Software Inc., Cleveland, $\mathrm{OH})$, using the DICOMRT protocol. Dose-volume histograms (DVHs) for PTV, CA, and spinal cord were calculated for all plans, and the mean DVH was evaluated based on the results. To evaluate the PTV dose coverage, the conformity index $(\mathrm{CI})$ and homogeneity index $(\mathrm{HI})$ were calculated for PTV under each plan [14,15]. CI is defined as follows:

$$
\mathrm{Cl}=\frac{\mathrm{PTV}_{\text {PIV }}{ }^{2}}{\mathrm{PTV} \times \mathrm{PIV}}
$$

, where PTV PIV is the PTV encompassed within PIV, which is the volume covered by the prescription isodose surface. HI is an indication of dose uniformity within PTV; a value of 1 indicates a uniform PTV dose distribution, an ideal value. $\mathrm{HI}$ is defined as follows:

$$
H \mathrm{I}=\frac{\mathrm{D}_{2}}{\mathrm{D}_{98}}
$$

, where $\mathrm{D}_{2}$ and $\mathrm{D}_{98}$ are the minimum doses received by $2 \%$ and $98 \%$ of PTV, respectively.

We calculated $V_{35}, V_{50}, V_{63}\left(V_{D}\right.$ : the percentage of the organ volume receiving D Gy or more), and maximum dose ( $\left.D_{\max }\right)$ for both CAs. The spinal cord dose was compared with the maximum dose.

To evaluate the treatment time efficacies of the four treatment methods, the planning and treatment times of each plan were compared. To provide an objective measurement, planning time only included the beam generation, optimization, and dose-calculation times, as performed by a senior dosimetrist with 10 years of experience in IMRT planning. The target and OAR-contouring times were excluded. Four sets of plan for each patient were generated continuously and without any interruption in the same manner. The treatment time only included practical treatment time, excluding the patient setup time. In other words, after patient setup verification was completed, the time between the first beam-on and the last beam-off was measured. The Wilcoxon signedrank test and the Bonferroni correction were used to compare the following differences: 2F-3DCRT versus TH-3DCRT, 3F-IMRT versus TH-3DCRT, and TH-IMRT versus TH3DCRT. Our statistical analysis was performed using SAS ver. 9.4 (SAS Institute Inc., Cary, NC). A 2-tailed p-value $<0.05$ was considered statistically significant. 

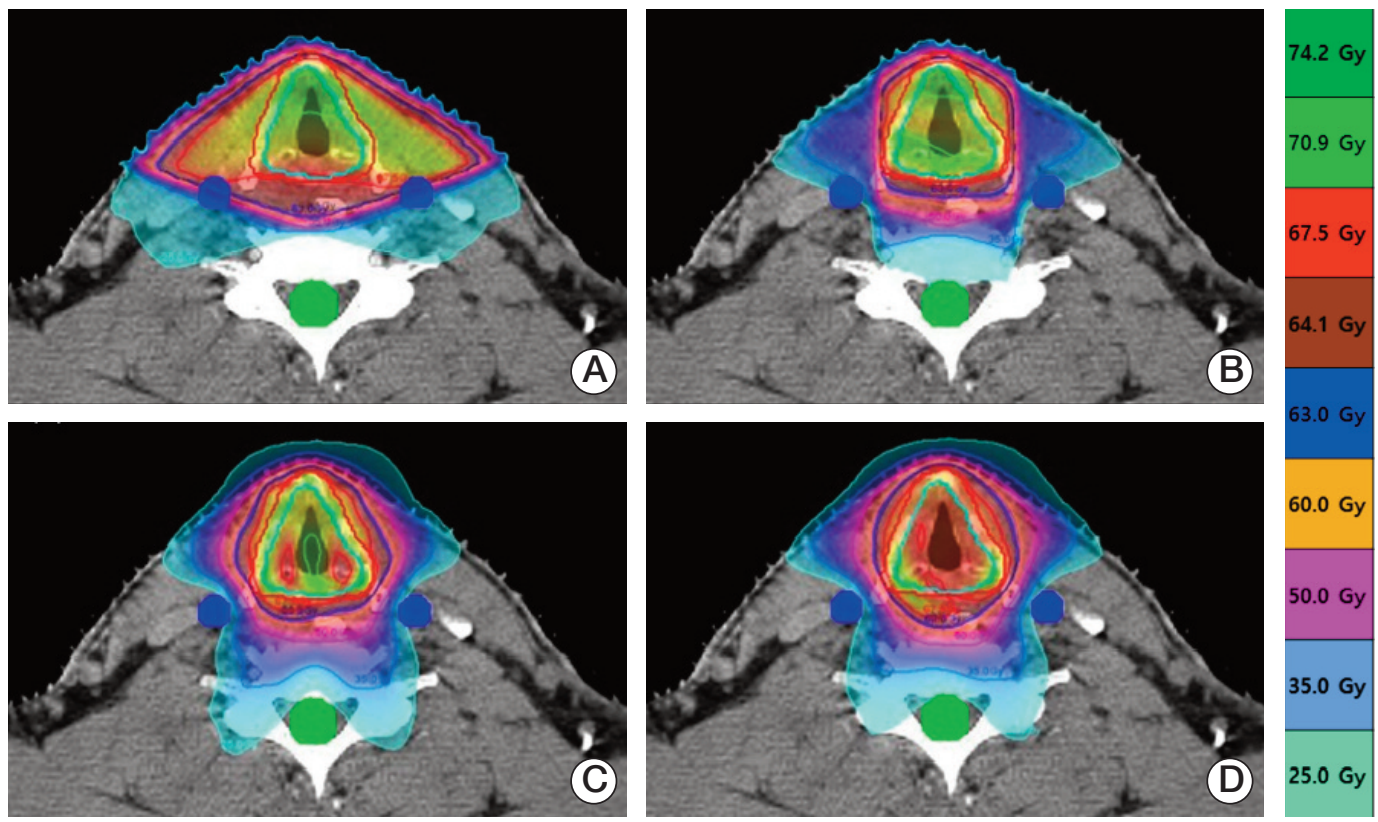

Fig. 1. An axial view of isodose distributions at the target center for each of the four treatment plans and a single representative patient. (A) Two-field 3-dimensional conformal radiotherapy (2F-3DCRT). (B) Three-field intensity-modulated radiation therapy. (C) TomoHelical IMRT. (D) TomoHelical 3DCRT. Under 2F-3DCRT, the high-dose region that received the prescription dose was widely distributed over the carotid artery. Under the other three plans, the high-dose region did not include the carotid artery.

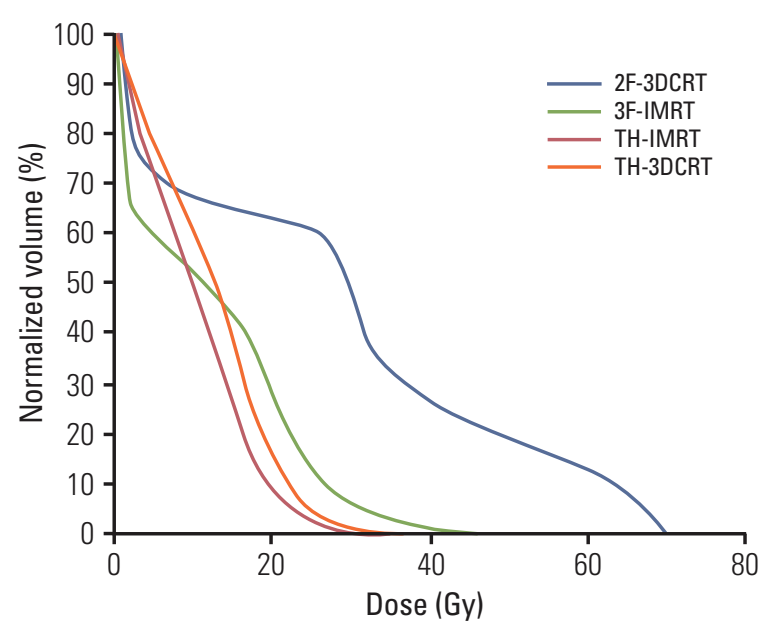

Fig. 2. This figure shows the mean dose-volume histogram (10 patients) for the carotid artery, including results for each of the four treatment plans that were investigated. In our treatment-planning simulations, the normalized volume of the carotid artery that received in excess of $30 \mathrm{~Gy}$ was the lowest using TomoHelical intensity-modulated radiation therapy (TH-IMRT), the second-lowest using TomoHelical 3-dimensional conformal radiotherapy (TH3DCRT), the third-lowest using 3-field IMRT (3F-IMRT), and the highest using 2-field 3DCRT (2F-3DCRT).

\section{Results}

\section{Plan comparison}

The 4 types of plans were associated with significantly different dose distributions for the same patients with EGC (Fig. 1). As viewed from the axial plane at the target center, unnecessary irradiation was observed on the left and right sides of the target under the 2F-3DCRT plan, as a consequence of the broad distribution of the regions receiving the prescribed dose, which was wider than observed under the other 3 plans. This resulted in a lower CI (median, 0.32; interquartile rage, 0.3 to 0.35 ) than was observed for the other plans (Table 2). Conformity improved in the following order: 3F-IMRT, TH-IMRT, and TH-3DCRT (Fig. 1). However, we found no statistically significant differences between the CIs associated with the plans ( $p>0.05$ ) (Table 2). The TH-3DCRT and TH-IMRT plans did not result in significantly different HIs. Further, the TH-3DCRT and 2F-3DCRT plans did not result in significantly different HIs. However, the 3F-IMRT plan resulted in a slightly higher HI than did the TH-3DCRT plan $(\mathrm{p}<0.05)$.

The CA was included within the volume that received the prescription dose under the 2F-3DCRT plan. Accordingly, 
Table 2. Comparisons of dosimetric characteristics and delivery efficiencies for different treatment techniques

\begin{tabular}{|c|c|c|c|c|c|c|c|}
\hline \multirow[b]{2}{*}{ Parameter } & \multirow[b]{2}{*}{ 2F-3DCRT } & \multirow[b]{2}{*}{ 3F-IMRT } & \multirow[b]{2}{*}{ TH-IMRT } & \multirow[b]{2}{*}{ TH-3DCRT } & \multicolumn{3}{|c|}{ p-value } \\
\hline & & & & & $\begin{array}{c}\text { 2F-3DCRT vs. } \\
\text { TH-3DCRT }\end{array}$ & $\begin{array}{c}\text { 3F-IMRT vs. } \\
\text { TH-3DCRT }\end{array}$ & $\begin{array}{c}\text { TH-IMRT vs. } \\
\text { TH-3DCRT }\end{array}$ \\
\hline \multicolumn{8}{|l|}{ PTV } \\
\hline CI & $\begin{array}{c}0.32 \\
(0.3-0.35)\end{array}$ & $\begin{array}{c}0.65 \\
(0.62-0.67)\end{array}$ & $\begin{array}{c}0.64 \\
(0.60-0.68)\end{array}$ & $\begin{array}{c}0.63 \\
(0.60-0.67)\end{array}$ & $0.01^{\mathrm{a})}$ & 0.83 & 0.39 \\
\hline $\mathrm{HI}$ & $\begin{array}{c}1.06 \\
(1.05-1.07)\end{array}$ & $\begin{array}{c}1.09 \\
(1.08-1.10)\end{array}$ & $\begin{array}{c}1.05 \\
(1.05-1.06)\end{array}$ & $\begin{array}{c}1.05 \\
(1.048-1.063)\end{array}$ & 0.39 & $0.01^{\text {a) }}$ & $>0.99$ \\
\hline \multicolumn{8}{|l|}{ Carotid artery } \\
\hline $\mathrm{V}_{35}(\%)$ & $\begin{array}{c}33.88 \\
(20.44-41.36)\end{array}$ & $\begin{array}{c}0.30 \\
(0.05-0.30)\end{array}$ & $\begin{array}{c}0.00 \\
(0.00-0.00)\end{array}$ & $\begin{array}{c}0.00 \\
(0.00-0.00)\end{array}$ & $0.01^{\mathrm{a})}$ & $0.01^{\mathrm{a})}$ & $>0.99$ \\
\hline $\mathrm{V}_{50}(\%)$ & $\begin{array}{c}18.48 \\
(9.01-25.15)\end{array}$ & $\begin{array}{c}0.00 \\
(0.00-0.00)\end{array}$ & $\begin{array}{c}0.00 \\
(0.00-0.00)\end{array}$ & $\begin{array}{c}0.00 \\
(0.00-0.00)\end{array}$ & $0.01^{\text {a) }}$ & $>0.99$ & $>0.99$ \\
\hline $\mathrm{V}_{63}(\%)$ & $\begin{array}{c}7.97 \\
(3.97-13.17)\end{array}$ & $\begin{array}{c}0.00 \\
(0.00-0.00)\end{array}$ & $\begin{array}{c}0.00 \\
(0.00-0.00)\end{array}$ & $\begin{array}{c}0.00 \\
(0.00-0.00)\end{array}$ & $0.01^{\text {a) }}$ & $>0.99$ & $>0.99$ \\
\hline$D_{\max }(\%)$ & $\begin{array}{c}69.82 \\
(69.23-70.41)\end{array}$ & $\begin{array}{c}38.71 \\
(35.55-43.52)\end{array}$ & $\begin{array}{c}29.23 \\
(26.37-32.28)\end{array}$ & $\begin{array}{c}30.31 \\
(27.53-34.15)\end{array}$ & $0.01^{\text {a) }}$ & $0.01^{\text {a) }}$ & 0.09 \\
\hline \multicolumn{8}{|l|}{ Spinal cord } \\
\hline$D_{\max }(\%)$ & $\begin{array}{c}2.58 \\
(2.38-3.55)\end{array}$ & $\begin{array}{c}28.05 \\
(27.03-28.77)\end{array}$ & $\begin{array}{c}24.86 \\
(23.48-27.52)\end{array}$ & $\begin{array}{c}26.76 \\
(24.76-29.42)\end{array}$ & $0.01^{\text {a) }}$ & 0.97 & $0.01^{\mathrm{a})}$ \\
\hline $\begin{array}{l}\text { Planning time } \\
\text { (min) }\end{array}$ & $\begin{array}{c}5.85 \\
(5.50-6.25)\end{array}$ & $\begin{array}{c}45.48 \\
(40.65-48.28)\end{array}$ & $\begin{array}{c}35.30 \\
(32.91-40.78)\end{array}$ & $\begin{array}{c}7.10 \\
(6.35-8.40)\end{array}$ & $0.01^{\text {a) }}$ & $0.01^{\text {a) }}$ & $0.01^{\text {a) }}$ \\
\hline $\begin{array}{l}\text { Delivery time } \\
\text { (min) }\end{array}$ & $\begin{array}{c}2.06 \\
(1.99-2.11)\end{array}$ & $\begin{array}{c}2.48 \\
(2.45-2.51)\end{array}$ & $\begin{array}{c}2.90 \\
(2.81-2.97)\end{array}$ & $\begin{array}{c}2.86 \\
(2.73-3.00)\end{array}$ & $0.01^{\text {a) }}$ & $0.01^{\text {a) }}$ & $>0.99$ \\
\hline $\begin{array}{l}\text { Pretreatment } \\
\text { dosimetric QA }\end{array}$ & $x$ & ० & ० & $x$ & - & - & - \\
\hline
\end{tabular}

Values are presented as median (interquartile range). 2F-3DCRT, 2-field 3-dimensional conformal radiotherapy; 3F-IMRT, 3-field intensity-modulated radiation therapy; TH-IMRT, TomoHelical IMRT; TH-3DCRT, TomoHelical 3DCRT; PTV, planning target volume; $\mathrm{CI}$, conformity index; $\mathrm{HI}$, homogeneity index; $\mathrm{V}_{\mathrm{D}}$, the percentage of the organ volume that received D Gy or more; $D_{\max }$ maximum dose; $\mathrm{QA}$, quality assurance. ${ }^{\mathrm{a})}$ Statistically significant.

the 2F-3DCRT plan was associated with a higher CA dose than the other 3 plans, under which the high-dose region did not include CA (Fig. 1). These results are reflected by the mean DVH for each plan (Fig. 2). $\mathrm{V}_{35}, \mathrm{~V}_{50}$, and $\mathrm{V}_{63}$ were also observed to be very high under the 2F-3DCRT plan, as compared with the other plans $(\mathrm{p}<0.05)$ (Table 2). Furthermore, there were no results indicating that the CA exceeded $V_{50}$ or $\mathrm{V}_{63}$ in the 3F-IMRT, TH-IMRT, and TH-3DCRT plans. There were no significant differences in $\mathrm{V}_{35}$ between TH-3DCRT and TH-IMRT ( $p>0.05)$. However, 3F-IMRT resulted in a slightly higher $\mathrm{V}_{35}$ than TH-3DCRT $(\mathrm{p}<0.05)$. Under the 2F-3DCRT plan, the median of the maximum CA doses (69.82 Gy; range, 69.23 to 70.41) exceeded the prescription dose. Further, 3F-IMRT resulted in a significant increase in this median of maximums compared to TH-IMRT and TH3DCRT $(p<0.05)$. Under each of the plans, the maximum dose to the spinal cord was below $45 \mathrm{~Gy}$.

\section{Efficiency}

Both 3F-IMRT and TH-IMRT required inverse planning, and their median planning times were 45.5 and 35.3 minutes, respectively. In contrast, 2F-3DCRT and TH-3DCRT did not require inverse planning, and their median planning times were only 5.8 and 7.1 minutes, respectively (Table 2). The TH-3DCRT median beam delivery time did not differ significantly from the TH-IMRT median beam delivery time, but was slightly longer than the 2F-3DCRT and 3F-IMRT median beam delivery times $(p<0.05)$. However, each of the differences was less than 1 minute. 


\section{Discussion}

RT with LOF has played an important role in the treatment of EGC for a long time. However, studies have reported that the excessive radiation dose received by CA can increase the risk of stroke due to the development of stenosis and thickening of the vessel wall [3,4]. Accordingly, there is growing interest in using the latest RT techniques to reduce the irradiation of the CA. There are several advantages to the complicated new treatment techniques; however, these techniques also have disadvantages, such as increased workloads and high probability of error. Further study is necessary to clarify the balance of these advantages and disadvantages. In particular, performing RT for EGC with a very low dose to $\mathrm{CA}$ results in both the deterioration of target coverage and rapid increase in workload. The exact dose-response relationship for CA irradiation remains unclear. However, studies have reported statistically significant thickening of the vessel wall for doses of more than 35-50 Gy [16]. Hence, we did not consider CA doses of less than $35 \mathrm{~Gy}$, while generating 4 different plans for this study.

Our study considered 2F-3DCRT, which provides a more efficient method of lowering the CA dose, as compared with the traditional LOF technique. When indirectly comparing our results with previous studies, we found that 2F-3DCRT provides better target dose conformity and CA sparing than traditional LOF [17]. In addition, 3-field (right anterior oblique, left anterior oblique, and an anterior field) 3DCRT shows a slightly better CA sparing than 2F-3DCRT [6]. In this study, however, 3DCRT resulted in a lower $\mathrm{CI}$ and a higher CA dose than the other three plans. For this reason, various forms of IMRT techniques for EGC RT have recently been introduced, such as volumetric-modulated arc therapy (VMAT). VMAT was not included in this study, but has recently been the subject of considerable attention [18]. When comparing previous investigations of various VMAT techniques with the results of this study, only full-arc VMAT offered a $\mathrm{V}_{35}$ that was similar to our findings for 3F-IMRT, TH-IMRT, and TH-3DCRT (a $\mathrm{V}_{35}$ that was close to 0).

As compared with 3DCRT, IMRT is generally associated with longer treatment planning times and treatment times because of its need for inverse planning and complex beam delivery $[19,20]$. In the present study, we found that the median beam delivery time was the shortest for 2F-3DCRT (2.06 minutes) and longest for TH-IMRT (2.9 minutes); however, the difference was less than 1 minute. The beam delivery time of full-arc VMAT was generally less than 2 minutes, although the results may vary with the type of equipment and the dose rate used [21]. Therefore, fullarc VMAT offers slightly shorter beam delivery times than the plans studied here. However, the difference is not particularly substantial from a clinical perspective. The treatment planning time of IMRT is much longer than that of 2F-3DCRT. Particularly, the treatment planning time of fullarc VMAT (which usually requires inverse planning for the full arc) is much longer than that of 3F-IMRT.

TH-3DCRT is a conformal arc technique that modifies the beam by opening the MLC in accordance to the shape of the TV in a helical mode (without beam intensity modulation). The treatment planning times and treatment times of TH-3DCRT are short (total of less than 30 minutes) because TH-3DCRT does not include intensity modulation and additional patient-specific pretreatment quality assurance is not required due to the simple treatment method. At our center, we perform dose verification of the absolute point dose using an ionization chamber and the relative dose distributions using Gafchromic film (EBT, ISP, Wayne, NJ) with a solid phantom before treatment for all IMRT sessions (Table 2). These procedures include measurements and analysis, and take about 2 hours. When TH-3DCRT was initially introduced, the exact same quality assurance procedures were performed as part of the commissioning process. However, TH-3DCRT has not been performed as a general 3D-CRT procedure after the correct delivery dose was confirmed. Therefore, the same-day treatment with a CT plan may also be worth considering.

Setup margin and motion are important factors for a successful treatment of EGC. The patient's larynx is reported to move $20-25 \mathrm{~mm}$ in the caudal-cranial direction and 3-8 $\mathrm{mm}$ in the anterior-posterior direction during swallowing $[13,22,23]$. However, the incidence and duration of swallowing are reported to be small in most patients. Hence, patients at our center receive thorough instructions not to swallow during treatment, and we try to shorten the treatment time as much as possible. Several previous studies reported that, when treating EGC with daily image-guided repositioning using a CTV-PTV margin of $1-2 \mathrm{~mm}$, there is a very small CTV dose reduction due to geometrical uncertainty, including set-up errors, respiratory movements, interfractional volumetric changes, and deformations that occur during treatment. Nonetheless, sparing of OAR remains unchanged $[12,24,25]$. The CTV-PTV margin added in this study was 3 $\mathrm{mm}$ in all directions, except posteriorly $(1 \mathrm{~mm})$. This was considered to be a safe margin for TH-3DCRT and TH-IMRT, which involve simultaneous megavoltage CT image-guidance and the use of a thermoplastic mask. 


\section{Conclusion}

In this study, we showed that TH-3DCRT provides many new benefits, excellent target dose coverage, and CA-sparing similar to that of the other two IMRT techniques. TH-3DCRT also offers high efficiency, short planning times, and short beam delivery times (similar to 3DCRT). In addition, pretreatment quality assurance is not required due to the simplicity of the treatment method. This study may serve as an effective guide for selecting the best-performing method of treatment for EGC that also requires the lowest workload. A suitable balance of performance and workload can be especially advantageous for busy clinics.

\section{Conflicts of Interest}

Conflict of interest relevant to this article was not reported.

\section{Acknowledgments}

This work was supported by the Technology Innovation Program, 10040362, Development of an integrated management solution for radiation therapy funded by the Ministry of Knowledge Economy (MKE, Korea) and by Samsung Medical Center grant (GFO1130081).

\section{References}

1. Teshima T, Chatani M, Inoue T. Radiation therapy for early glottic cancer (T1N0M0): II. prospective randomized study concerning radiation field. Int J Radiat Oncol Biol Phys. 1990;18:119-23.

2. Chatani M, Matayoski Y, Masaki N, Teshima T, Inoue T. Radiation therapy for early glottic carcinoma (T1N0M0): the final results of prospective randomized study concerning radiation field. Strahlenther Onkol. 1996;172:169-72.

3. Brown PD, Foote RL, McLaughlin MP, Halyard MY, Ballman $\mathrm{KV}$, Collie AC, et al. A historical prospective cohort study of carotid artery stenosis after radiotherapy for head and neck malignancies. Int J Radiat Oncol Biol Phys. 2005;63:1361-7.

4. Smith GL, Smith BD, Buchholz TA, Giordano SH, Garden AS, Woodward WA, et al. Cerebrovascular disease risk in older head and neck cancer patients after radiotherapy. J Clin Oncol. 2008;26:5119-25.

5. Dorresteijn LD, Kappelle AC, Boogerd W, Klokman WJ, Balm $\mathrm{AJ}$, Keus RB, et al. Increased risk of ischemic stroke after radiotherapy on the neck in patients younger than 60 years. J Clin Oncol. 2002;20:282-8.

6. Chera BS, Amdur RJ, Morris CG, Mendenhall WM. Carotidsparing intensity-modulated radiotherapy for early-stage squamous cell carcinoma of the true vocal cord. Int J Radiat Oncol Biol Phys. 2010;77:1380-5.

7. Rosenthal DI, Fuller CD, Barker JL Jr, Mason B, Garcia JA, Lewin JS, et al. Simple carotid-sparing intensity-modulated radiotherapy technique and preliminary experience for T1-2 glottic cancer. Int J Radiat Oncol Biol Phys. 2010;77:455-61.

8. Gomez D, Cahlon O, Mechalakos J, Lee N. An investigation of intensity-modulated radiation therapy versus conventional two-dimensional and 3D-conformal radiation therapy for early stage larynx cancer. Radiat Oncol. 2010;5:74.

9. Chatterjee S, Guha S, Prasath S, Mallick I, Achari R. Carotid sparing hypofractionated tomotherapy in early glottic cancers: refining image guided IMRT to improve morbidity. J Cancer Res Ther. 2013;9:452-5.

10. Cheung K. Intensity modulated radiotherapy: advantages, limitations and future developments. Biomed Imaging Interv J. 2006;2:e19.

11. Court LE, Seco J, Lu XQ, Ebe K, Mayo C, Ionascu D, et al. Use of a realistic breathing lung phantom to evaluate dose delivery errors. Med Phys. 2010;37:5850-7.

12. Osman SO, de Boer HC, Astreinidou E, Gangsaas A, Heijmen $\mathrm{BJ}$, Levendag PC. On-line cone beam CT image guidance for vocal cord tumor targeting. Radiother Oncol. 2009;93:8-13.

13. van Asselen B, Raaijmakers $\mathrm{CP}$, Lagendijk JJ, Terhaard $\mathrm{CH}$. Intrafraction motions of the larynx during radiotherapy. Int J Radiat Oncol Biol Phys. 2003;56:384-90.

14. Paddick I. A simple scoring ratio to index the conformity of radiosurgical treatment plans. Technical note. J Neurosurg. 2000;93 Suppl 3:219-22.

15. Prasana Sarathy N. Evaluation of three dimensional conformal versus field in field forward IMRT planning for intact breast irradiation. Mumbai: Association of Medical Physicists of India; 2008. p. 32-3.

16. Martin JD, Buckley AR, Graeb D, Walman B, Salvian A, Hay $\mathrm{JH}$. Carotid artery stenosis in asymptomatic patients who have received unilateral head-and-neck irradiation. Int J Radiat Oncol Biol Phys. 2005;63:1197-205.

17. Ki YK, Kim WT, Nam JH, Kim DH, Lee JH, Park D, et al. Treatment planning for minimizing carotid artery dose in the radiotherapy of early glottic cancer. J Korean Soc Ther Radiol Oncol. 2011;29:115-20.

18. Riegel AC, Antone J, Schwartz DL. Comparative dosimetry of volumetric modulated arc therapy and limited-angle static intensity-modulated radiation therapy for early-stage larynx cancer. Med Dosim. 2013;38:66-9.

19. Moran JM, Dempsey M, Eisbruch A, Fraass BA, Galvin JM, 
Ibbott GS, et al. Safety considerations for IMRT: executive summary. Pract Radiat Oncol. 2011;1:190-5.

20. Fraass BA, Marks LB, Pawlicki T; ASTRO Multidisciplinary Quality Assurance Subcommittee. Commentary: safety considerations in contemporary radiation oncology: introduction to a series of ASTRO safety white papers. Pract Radiat Oncol. 2011;1:188-9.

21. Bertelsen A, Hansen CR, Johansen J, Brink C. Single arc volumetric modulated arc therapy of head and neck cancer. Radiother Oncol. 2010;95:142-8.

22. Dantas RO, Kern MK, Massey BT, Dodds WJ, Kahrilas PJ, Brasseur JG, et al. Effect of swallowed bolus variables on oral and pharyngeal phases of swallowing. Am J Physiol. 1990; 258(5 Pt 1):G675-81.

23. Leonard RJ, Kendall KA, McKenzie S, Goncalves MI, Walker A. Structural displacements in normal swallowing: a videofluoroscopic study. Dysphagia. 2000;15:146-52.

24. Osman SO, Astreinidou E, Levendag PC, Heijmen BJ. Impact of geometric variations on delivered dose in highly focused single vocal cord IMRT. Acta Oncol. 2014;53:278-85.

25. Levendag PC, Teguh DN, Keskin-Cambay F, Al-Mamgani A, van Rooij P, Astreinidou E, et al. Single vocal cord irradiation: a competitive treatment strategy in early glottic cancer. Radiother Oncol. 2011;101:415-9. 